\title{
Investigation of the Relationship between Malignant Tumors Originated from Peritoneal and Müllerian Epithelium with the Primary and Secondary Müllerian Systems - Immunohistochemical Analysis with Six Markers
}

\author{
Ayse Ciftci ${ }^{1}$, Handan Aker ${ }^{2}$ and Hatice Ozer ${ }^{2 *}$ \\ ${ }^{1}$ Department of Pathology, Ankara Numune Education and Research Hospital, Ankara, Turkey \\ ${ }^{2}$ Department of Pathology, Faculty of Medicine, Cumhuriyet University, Sivas, Turkey
}

*Corresponding author: Hatice Ozer, MD, Associate Professor, Department of Pathology, Faculty of Medicine, Cumhuriyet University, Sivas, Turkey, Tel: 0-536-7159321,Fax: 903462581304,E-mail: haticozer@gmail.com

\begin{abstract}
The origin of ovarian epithelial tumors is still indistinct. Müllerian and coelomic hypothesis are controversial. Recently, there is also a small number of reports that tubo-peritoneal junction may play an important role in the development of tubo-ovarian tumors. Therefore, we aimed to investigate the relationship between tumors originating from Mullerian epithelium and peritoneal mesothelium, in other words primary and secondary Müllerian systems.
\end{abstract}

Seventy malignant tumors originating from the Müllerian epithelium and peritoneal mesothelium were included in the study. Tumors were analysed immunohistochemically for PAX8, WT1, CD56, Ber-EP4, ER and desmin expression. We evaluated the study based on three phases. Firstly, we investigated immunohistochemical stainings of different tumor types in different locations. Secondly, we assessed immunohistochemical characteristics according to their histologicals subtypes, and finally their relationship with primary or secondary Müllerian systems.

As a result different immunohistochemical features were detected between both localization and histological types of these tumor groups.

Absence of immunoreactivity of PAX8, WT1 and CD56 in endometrial serous carcinomas and, the presence of high immunoreactivity in ovarian and tubal serous carcinomas was considered to be an indicator of different origins of these tumors as primary and secondary Müllerian systems. It also seems possible that tubo-peritoneal junction could be the junction between primary and secondary Müllerian systems and, may play an important role in the development of tubo-ovarian tumors.

\section{Keywords}

Müllerian system, Coelomic epithelium, Mesothelium, Ovarian surface epithelium, Malignant tumors, Immunohistochemistry

\section{Introduction}

One of the most curious features of ovarian epithelial neoplasms is their histogenesis. The true source of origin of these normal cell types giving rise to these tumors is unclear. In some of these epithelial ovarian tumors, the cell morphology is indistinguishable from neoplasms arising from those organs of the female genital tract that are embryologically derived from Müllerian ducts just as the serous, the mucinous and the endometrioid types resemble the neoplasms of fallopian tubes, endocervix and endometrium, respectively. Besides, in most cases, primary peritoneal serous carcinomas (PSC), peritoneal malignant mesotheliomas (PMM) and also metastatic ovarian tumors showing similarity with ovarian serous carcinomas (OSC) have difficulties in discriminating. Since the treatment approaches are different in these tumor types, it is very important to achieve the definite diagnosis [1-3]. Lauchlan used the term secondary Müllerian system (SMS) to designate the structures lined by Müllerian epithelium found outside the fallopian tubes, uterus, and cervix while fallopian tubes, uterus, cervix and upper part of vagina derived from Müllerian ducts are

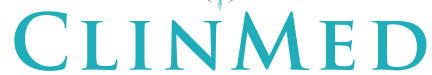

INTERNATIONAL LIBRARY
Received: November 27, 2016; Accepted: April 17, 2017; Published: April 20, 2017

Copyright: (C) 2017 Ciftci A, et al. This is an open-access article distributed under the terms of the Creative Commons Attribution License, which permits unrestricted use, distribution, and reproduction in any medium, provided the original author and source are credited. 
called as primary Müllerian system (PMS) [4]. So, SMS is seemed to be the source of paraovarian and paratubal cysts, rete ovarii, endosalpigiosis, endometriosis and endocervicosis. Although ovary is not originated from PMS, it can contain SMS structures such as Müllerian duct remnants, rete ovarii, endosalpingiosis, endometriosis, and endocervicosis [4]. The coelomic epithelium ultimatelly links to the extraovarian mesothelium that is the region of tubo-peritoneal junction (TPJ) [5]. Since the junctions between different types of epithelia are designated as hot spots for carcinogenesis, the junction of the peritoneal mesothelium with the fallopian tubal epithelium may play a role in the development of these tumors [5]. The role of epithelial junctions, notably the cervical squamocolumnar, gastroesophageal, and anorectal junctions in neoplasia was well recognized but the junction of the columnar tubal epithelium with the mesothelial serosa on the fimbriae has rarely been mentioned in the literature. If regional junctions is important in carcinogenesis then the TPJ should be of particular interest [5]. On the other hand, ovarian surface epithelium is considered as modified pelvic mesothelium, originates from coelomic mesoderm, and is reported to have in both mesenchymal and epithelial properties unlike the tubal and the endometrial epithelium developing from PMS. These spesific features of ovarian surface epithelium may partly explain the different characteristics of the tumors with similar morphology originated from PMS and SMS [3,6-11]. In this study our aim is to investigate the relationship between tumors originating from Müllerian epithelium and peritoneal mesothelium, and also to contribute in differential diagnosis of these tumors by using six immunohistochemical (IHC) markers.

\section{Materials and Methods}

Cases that were diagnosed between 2005 and 2011 and diagnosed as OSC and tubal serous carcinomas (TSC) from the ovary and tuba; endometrioid carcinoma (EEC) and serous carcinoma (ESC) from the endometrium; PSC and PMM from the peritoneum were obtained from the archival records in the Department of Pathology at Cumhuriyet University Hospital, Sivas, Turkey. Patients age and tumor localizations were retrieved from the medical records. The histologic diagnosis of each tumor was confirmed on the hematoxylin and eosin stained sections. Twenty cases of OSC, 31 cases of EEC, 4 cases of ESC, 8 cases of TSC, 4 cases of PMM and 3 cases of PSC composed of totally 70 cases were included in this study. Cases in the study were divided into three groups as serous carcinoma ( $\mathrm{SC}$ ), endometrioid carcinoma (EC), and malign mesothelioma (MM) according to their histologic types. Moreover, cases were also divided into two groups as PMS tumors which were located distal to the TPJ and assumed to be derived from Müllerian ductus, and SMS tumors which were located proximal to the TPJ and assumed to be derived from modified mesothelium. Thus, EEC and ESC cases were evaluated as PMS tumors; OSC, TSC, PSC and PMM cases were considered as SMS tumors. H\&E stained sections of the cases re-evaluated and the slides were selected from parafin blocks containing representative tumor without necrosis. Sections of $3 \mu \mathrm{m}$ thickness were taken from these parafin blocks to Poly-L-Lysine covered slides. All of the cases were analysed IHC with PAX8 (a sensitive and spesific marker for Müllerian originated tumors), WT1 (a marker for both Müllerian and mesothelial origin), CD56 (a newly defined and very sensitive marker for ovarian sex cord stromal tumors), Ber-EP4 (an epithelial marker), ER (a marker having an important role in development and treatment of gynecological tumors), and desmin (a mesenchymal marker). Avidin-biotin peroxidase IHC method was applied to selected sections using a panel of antibodies to PAX8 (Cell Marque rabbit policlonal antibody, Clone 112970.2A, 1:50 dilution ratio), WT1 (Dako, Mouse Monoclonal Antibody, Clone 6f-H2, 7.0 $\mathrm{ml}$ ready to use form), CD56 (Thermo Scientific, Mouse Monoclonal Antibody, Clone 123C3.D5, $7.0 \mathrm{ml}$ ready to use form), Ber-EP4 (Biocare Medikal, Mouse Monoclonal Antibody, Clone Ber-EP4, $7.0 \mathrm{ml}$ ready to use form), ER (Dako, Rabbit monoclanal antibody, clone EP1, $7.0 \mathrm{ml}$ ready to use form) ve desmin (Scytek, Mouse Monoclonal Antibody, Clone D33, $7.0 \mathrm{ml}$ ready to use form).

\section{Immunohistochemical evaluation}

Nuclear staining for PAX8, WT1 and ER; membranous staining for Ber-EP4 and CD56; cytoplasmic staining for desmin were accepted as positive. Furthermore, positive control slides were prepared for each IHC stain. Immunoreactivity was scored semiquantitively based on intensity of staining and percentage of immunoreactive area [12]. The intensity of stained cells was scored as 0 ( no staining), 1+ (weak staining), 2+ (moderate staining), $3+$ (strong staining). The percentage of immunoreactive area was scored as 1 (less than 10\%), 2 (10\% to 50\%), $3(52 \%$ to $80 \%$ ), and 4 (more than $80 \%$ ). For each case, the values of the two parameters (predominant intensity and percentage of the positive cells) were multiplied, resulting in scores from 0 to 12 . Final score was classified as negative or low reactive (score $0-1$ ), moderate (scores 2-6), and high (scores 8-12). For the values obtained for each antibody, cases were again scored by giving numerical values as negative or low (1), moderate (2), and high (3) for statistical data.

\section{Statistical analysis}

Data were installed to SPSS 14.0 (SPSS Inc, Chicago, III, USA) program. Kruskal-Wallis, Mon-Whitney $U$ and Chisquare tests were used to evaluate the data analysis. Values with $p<0.05$ were accepted as statistically significant.

\section{Results}

Of the 70 cases included in the study, 20 (29\%) cases were OSC, $8(11 \%)$ cases were TSC, $4(6 \%)$ cases were ESC, 31 (44\%) cases were EEC, 3 (4\%) cases were PSC, 
and $4(6 \%)$ cases were PMM. The age of the youngest in the overall evaluation of patients was 34 and the oldest was 85-years-old and the average age was $60.5 \pm 19.9$. The mean age, the standard deviation, and the ages of the youngest and the oldest of the tumor groups were shown in Table 1. Statistical analysis of the IHC results of all tumors belonging to groups PAX8, WT1, CD56, BerEP4, ER, and desmin expressions was shown in Table 2. According to the histological types we divided the tumors into three groups as SC, EC and MM, tumors of the 70 cases were categorized as 35 cases of (50\%) SC, 31 cases of (44.3\%) EC, and 4 cases of (5.7\%) MM. Of the SCs, 20 tumors were localized at the ovary, 8 at the fallopian tube, 4 at the endometrium, and 3 in the peritoneal cavity. Immunoreactive histological types stained with markers were shown on Table 3, Figure 1, Figure 2, Figure 3 , Figure 4 and Figure 5. Tumors which were divided into two groups as PMS and SMS according to origin and their IHC staining characteristics were shown in Table 4. Each group was consisted of 35 patients $(50 \%)$. In tumors of the PMS group, 31 (88.6\%) were EEC, 4 (11.4\%) were ESC; and in tumors of the SMS group, 8 (22.9\%) were TSC, $20(57.1 \%)$ were OSC, $3(8.6 \%)$ were PSC, and $4(11.4 \%)$ were PMM. As a result values of six different markers in different six tumor groups were found significant $(p<$ 0.05 ) except desmin. Epithelial marker was all reactive in tumors except peritoneal malignant mesotheliomas whereas no expression was detected with mesenchymal

Table 1: The age distribution of cases.

\begin{tabular}{|l|l|l|l|l|l|l|l|}
\hline & OSC & TSC & ESC & EEC & PSC & PMM & Total \\
\hline N & 20 & 8 & 4 & 31 & 3 & 4 & 70 \\
\hline Mean & 57.7 & 6.5 & 74.5 & 58.9 & 74.3 & 58.3 & 60.5 \\
\hline SD $\mathbf{S}$ & 12.3 & 13.2 & 8.2 & 10.8 & 12.9 & 10.5 & 19.9 \\
\hline Oldest & 80 & 76 & 85 & 83 & 85 & 72 & 85 \\
\hline Youngest & 34 & 42 & 65 & 37 & 60 & 45 & 34 \\
\hline
\end{tabular}

*SD: Standard deviation.

Table 2: Statistical analysis of the immunohistochemical results for all groups.

\begin{tabular}{|c|c|c|c|c|c|c|}
\hline Groups & PAX8 & WT1 & CD56 & Ber-EP4 & ER & Desmin \\
\hline & $\begin{array}{l}\text { Mean } \\
\text { median } \\
\text { min-max }\end{array}$ & $\begin{array}{l}\text { Mean } \\
\text { median } \\
\text { min-max }\end{array}$ & $\begin{array}{l}\text { Mean } \\
\text { median } \\
\text { min-max }\end{array}$ & $\begin{array}{l}\text { Mean } \\
\text { median } \\
\text { min-max }\end{array}$ & $\begin{array}{l}\text { Mean } \\
\text { median } \\
\text { min-max }\end{array}$ & $\begin{array}{l}\text { Mean } \\
\text { median } \\
\text { min-max }\end{array}$ \\
\hline \multirow{3}{*}{ osc } & 2.20 & 2.20 & 1.45 & 2.35 & 2.50 & 1.00 \\
\hline & 2.00 & 2.00 & 1.0 & 2.50 & 3.00 & 1.00 \\
\hline & $(1-3)$ & $(1-3)$ & $(1-3)$ & $(1-3)$ & $(1-3)$ & $(1-1)$ \\
\hline \multirow{3}{*}{ TSC } & 2.00 & 2.37 & 1.37 & 2.00 & 2.12 & 1.00 \\
\hline & 2.00 & 2.50 & 1.00 & 2.00 & 2.30 & 1.00 \\
\hline & $(1-3)$ & $(1-3)$ & $(1-2)$ & $(1-3)$ & $(1-3)$ & $(1-1)$ \\
\hline \multirow{3}{*}{ ESC } & 1.00 & 1.00 & 1.00 & 2.25 & 1.75 & 1.00 \\
\hline & 1.00 & 1.00 & 1.00 & 2.00 & 2.00 & 1.00 \\
\hline & $(1-1)$ & $(1-1)$ & $(1-1)$ & $(2-3)$ & $(1-2)$ & $(1-1)$ \\
\hline \multirow{3}{*}{ EEC } & 1.51 & 1.00 & 1.06 & 2.22 & 2.48 & 1.00 \\
\hline & 1.00 & 1.00 & 1.00 & 2.00 & 3.00 & 1.00 \\
\hline & $(1-3)$ & $(1-1)$ & $(1-2)$ & $(1-3)$ & $(1-3)$ & $(1-1)$ \\
\hline \multirow{3}{*}{ PSC } & 2.00 & 1.33 & 1.00 & 3.00 & 2.66 & 1.00 \\
\hline & 2.00 & 1.00 & 1.00 & 3.00 & 3.00 & 1.00 \\
\hline & $(1-3)$ & $(1-1)$ & $(1-1)$ & $(1-3)$ & $(2-3)$ & $(1-1)$ \\
\hline \multirow{3}{*}{ PMM } & 1.25 & 2.00 & 1.00 & 1.00 & 1.00 & 1.00 \\
\hline & 1.00 & 2.00 & 1.00 & 1.00 & 1.00 & 1.00 \\
\hline & $(1-1)$ & $(1-3)$ & $(1-1)$ & $(1-1)$ & $(1-1)$ & $(1-1)$ \\
\hline \multirow{2}{*}{ Result } & $\mathrm{KW}=17.97$ & $\mathrm{KW}=77.70$ & $K W=13.39$ & $\mathrm{KW}=15.39$ & $\mathrm{KW}=14.22$ & - \\
\hline & ${ }^{*} p=0.03$ & $p=0.001$ & $p=0.020$ & ${ }^{*} p=0.009$ & ${ }^{*} p=0.014$ & - \\
\hline
\end{tabular}

min-max: minimum-maximum, 1: low or negative, 2 : medium, 3 : high, "significant if $p<0.05$.

Table 3: The distribution of cases according to tumor type and their immunoreactive features**.

\begin{tabular}{|c|c|c|c|c|c|c|c|c|c|c|c|c|}
\hline \multirow{2}{*}{ Type } & \multirow{2}{*}{ n } & \multirow{2}{*}{$\%$} & \multicolumn{2}{|c|}{ PAX8 } & \multicolumn{2}{|c|}{ WT1 } & \multicolumn{2}{|c|}{ CD56 } & \multicolumn{2}{|c|}{ BER-EP4 } & \multicolumn{2}{|c|}{ ER } \\
\hline & & & $\mathrm{n}$ & $\%$ & $\mathbf{N}$ & $\%$ & $n$ & $\%$ & $\mathrm{n}$ & $\%$ & $\mathrm{n}$ & $\%$ \\
\hline SC & 35 & 50.0 & 25 & 71.4 & 26 & 74.2 & 11 & 31.4 & 30 & 85.7 & 28 & 80.0 \\
\hline EC & 31 & 44.3 & 13 & 42.0 & 0 & 0.0 & 2 & 6.5 & 28 & 90.4 & 26 & 83.9 \\
\hline MM & 4 & 5.7 & 1 & 25.0 & 2 & 50.0 & 0 & 0.0 & 0 & 0.0 & 0 & 0.0 \\
\hline Total & 70 & 100.0 & 39 & 55.7 & 28 & 40.0 & 13 & 18.6 & 58 & 82.9 & 54 & 77.1 \\
\hline \multirow[t]{2}{*}{ Result } & & & \multicolumn{2}{|c|}{$X^{2}=7.41$} & \multicolumn{2}{|c|}{$X^{2}=37.97$} & \multicolumn{2}{|c|}{$X^{2}=7.74$} & \multicolumn{2}{|c|}{$X^{2}=22.75$} & \multicolumn{2}{|c|}{$X^{2}=14.45$} \\
\hline & & & \multicolumn{2}{|c|}{${ }^{*} p=0.025$} & \multicolumn{2}{|c|}{${ }^{*} p=0.001$} & \multicolumn{2}{|c|}{${ }^{*} p=0.021$} & \multicolumn{2}{|c|}{$p=0.001$} & \multicolumn{2}{|c|}{${ }^{*} p=0.001$} \\
\hline
\end{tabular}

"significant if $p<0.05$; *None of the tumors react with desmin. 

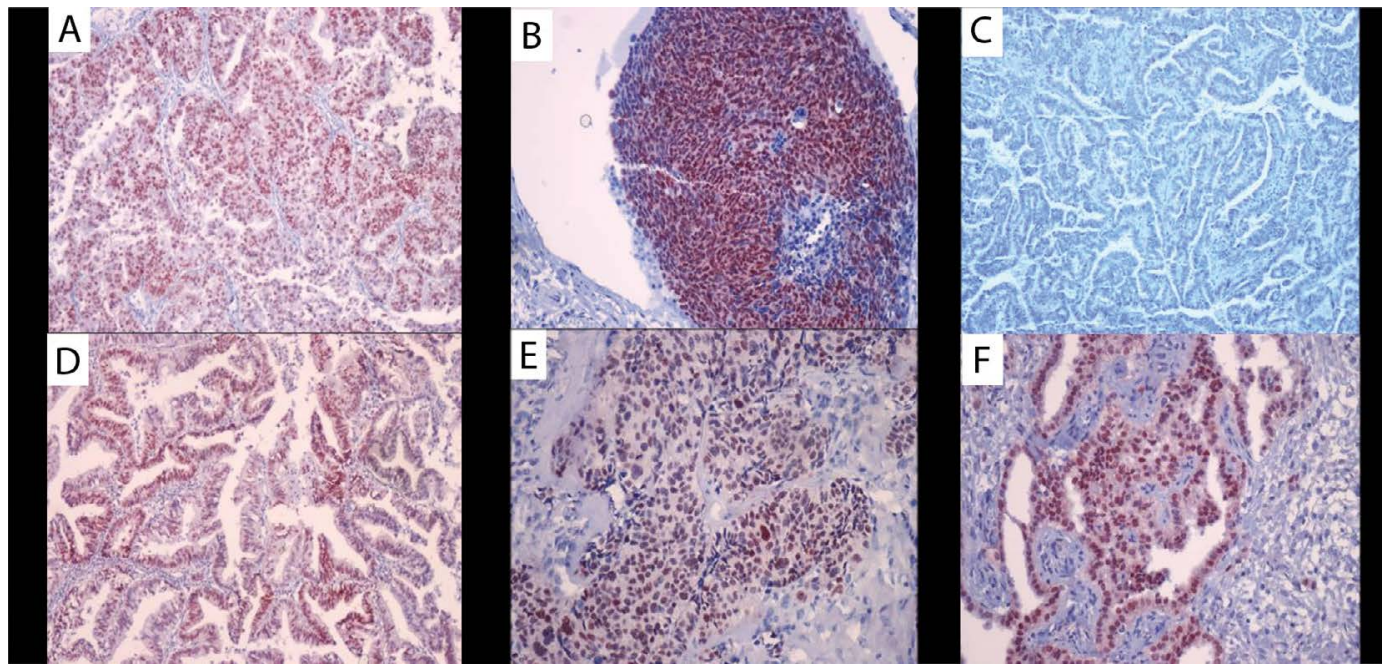

Figure 1: Immunohistochemical staining for PAX8.

A) score 3, in OSC (X 100); B) score 3, in TSC (X 200); C) score 1, in ESC (X 100); D) score 3, in EEC (X 100); E) score 3, in PSC (X 200) and F) score 2, in PMM (X 200).
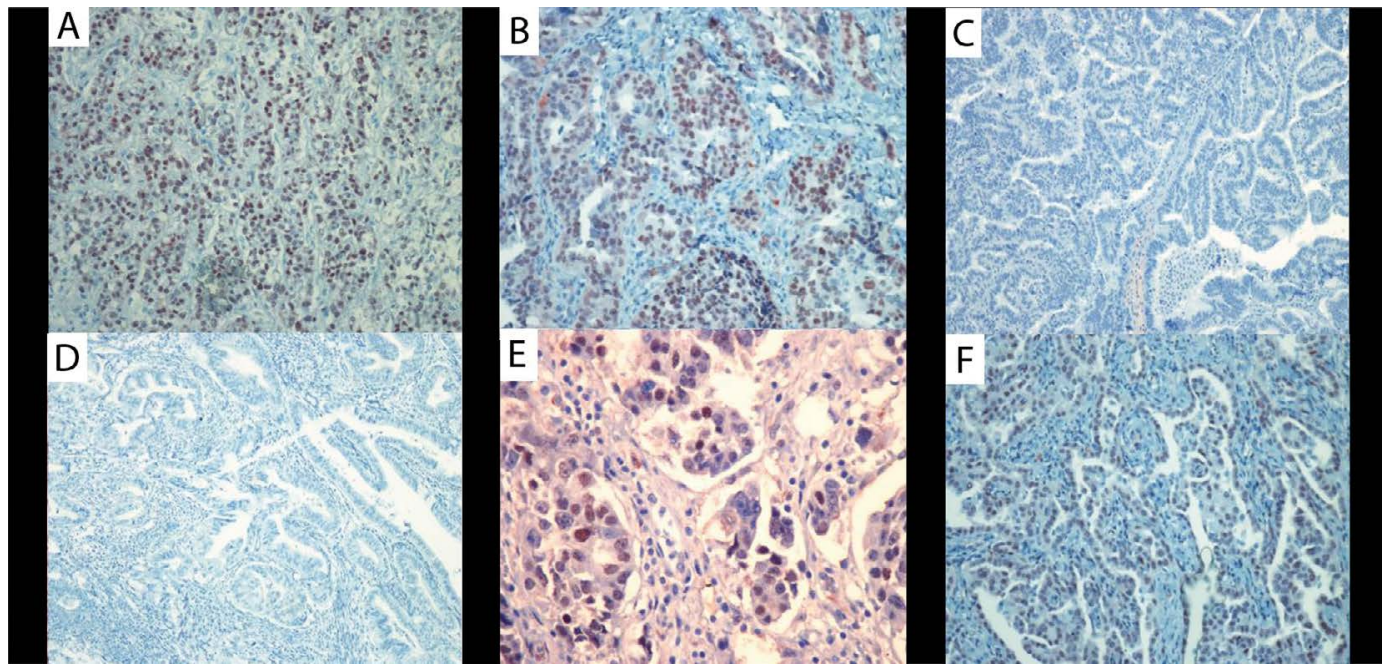

Figure 2: Immunohistochemical staining for WT1.

A) score 3, in OSC (X 200); B) score 3, in TSC (X 200); C) score 1, in ESC (X 100); D) score 1, in EEC (X 100); E) score 2, in PSC (X 400) and F) score 3, in PMM (X 200).
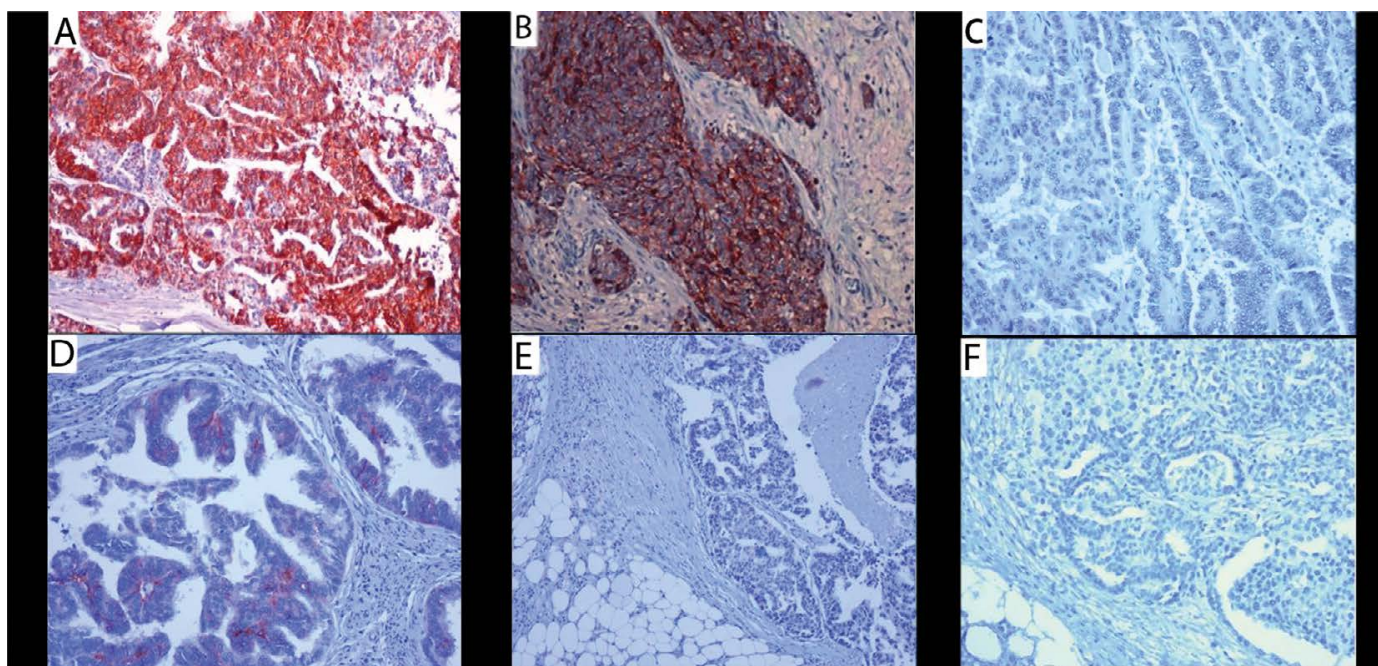

Figure 3: Immunohistochemical staining for CD56.

A) score 3, in OSC (X 200); B) score 2, in TSC (X 400); C) score 1, in ESC (X 200); D) score 2, in EEC (X 400); E) score 1, in PSC (X 100) and F) score 1 , in PMM (X 200). 


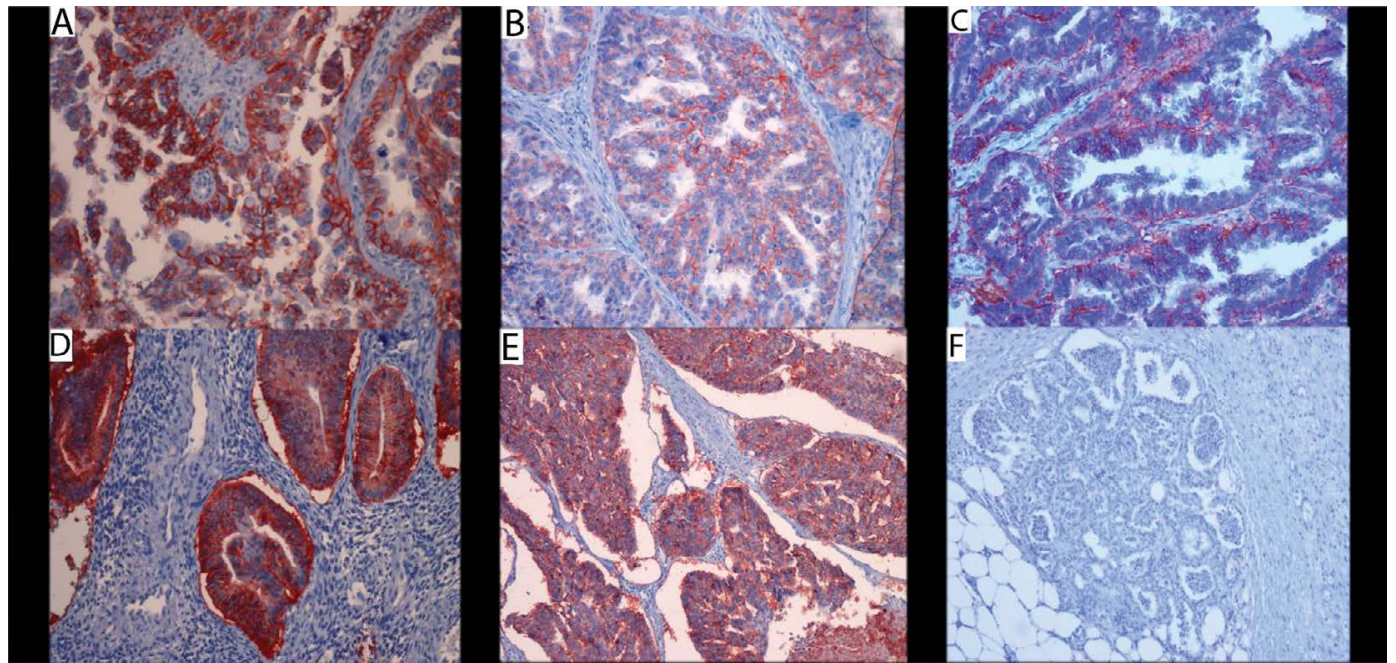

Figure 4: Immunohistochemical staining for Ber-EP4.

A) score 3, in OSC (X 200); B) score 3, in TSC (X 200); C) score 2, in ESC (X 400); D) score 3, in EEC (X 200); E) score 3, in PSC (X 200) and F) score 1, in PMM (X 100).
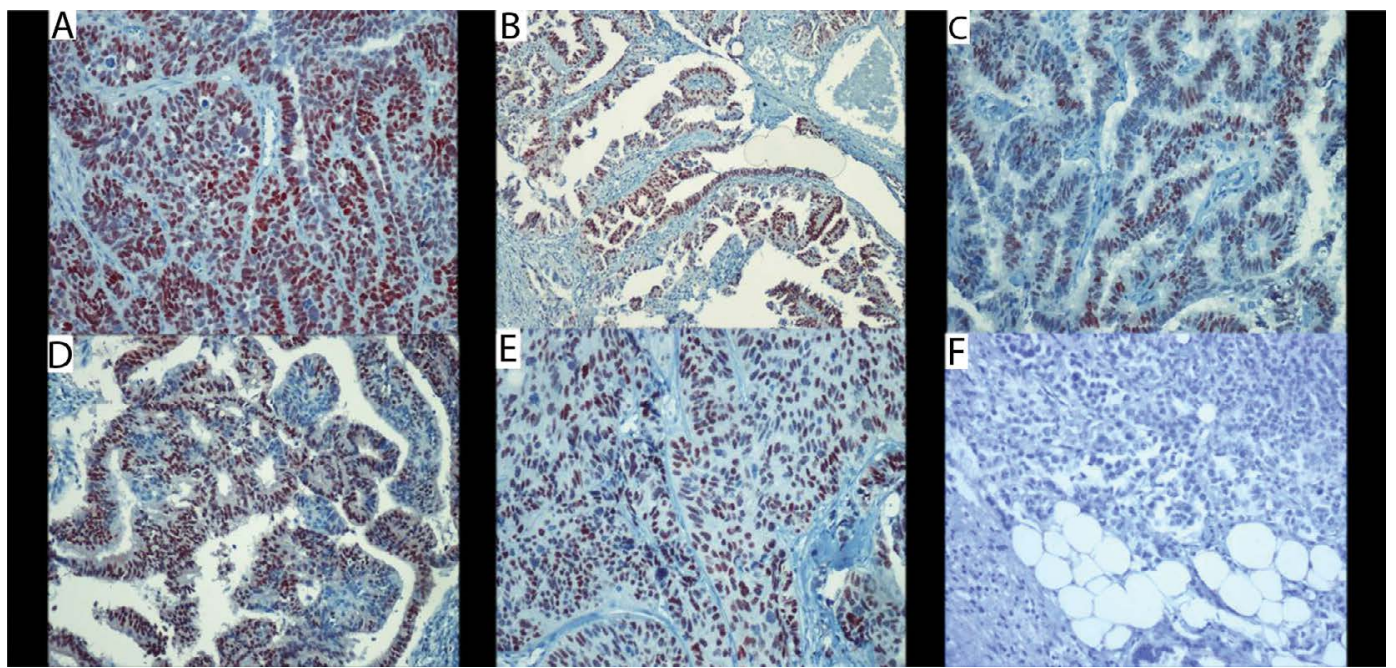

Figure 5: Immunohistochemical staining for ER.

A) score 3, in OSC (X 200); B) score 3, in TSC (X 100); C) score 2, in ESC (X 200); D) score 3, in EEC (X 200); E) score 3, in PSC (X 200) and F) score 1, in PMM (X 400).

Table 4: Distribution of cases according to PMS and SMS and their immunoreactive features ${ }^{\star *}$.

\begin{tabular}{|c|c|c|c|c|c|c|c|c|c|c|c|c|}
\hline & \multirow{2}{*}{\multicolumn{2}{|c|}{$\begin{array}{l}\text { PAX8 } \\
13(37.1 \%)\end{array}$}} & \multirow{2}{*}{\multicolumn{2}{|c|}{$\begin{array}{l}\text { WT1 } \\
0(0)\end{array}$}} & \multicolumn{2}{|c|}{ CD56 } & \multicolumn{2}{|c|}{ Ber-EP4 } & \multicolumn{2}{|l|}{ ER } & \multicolumn{2}{|c|}{ Total } \\
\hline \multirow{2}{*}{$\begin{array}{l}\text { PMS } \\
\text { EEC }\end{array}$} & & & & & \multicolumn{2}{|c|}{$2(5.7 \%)$} & \multicolumn{2}{|c|}{32 (91.4\%) } & \multicolumn{2}{|c|}{$29(82.9 \%)$} & \multicolumn{2}{|c|}{$35(100 \%)$} \\
\hline & 13 & $41.9 \%$ & \multicolumn{2}{|c|}{0} & 2 & $6.5 \%$ & 28 & $90.3 \%$ & 26 & $83.9 \%$ & 31 & $88.6 \%$ \\
\hline ESC & 0 & $0 \%$ & 0 & $0 \%$ & 0 & $0 \%$ & 4 & $100 \%$ & 3 & $75 \%$ & 4 & $11.4 \%$ \\
\hline SMS & \multicolumn{2}{|c|}{$26(74.3 \%)$} & \multicolumn{2}{|c|}{$28(80 \%)$} & \multicolumn{2}{|c|}{$11(31.4 \%)$} & \multicolumn{2}{|c|}{$26(74.3 \%)$} & \multicolumn{2}{|c|}{25 (71.4\%) } & \multicolumn{2}{|c|}{$35(100 \%)$} \\
\hline TSC & 5 & $62.5 \%$ & 7 & $87.5 \%$ & 3 & $37.5 \%$ & 6 & $75 \%$ & 5 & $62.5 \%$ & 8 & $22.9 \%$ \\
\hline OSC & 18 & $90 \%$ & 18 & $90 \%$ & 8 & $40 \%$ & 17 & $85 \%$ & 17 & $85 \%$ & 20 & $57.1 \%$ \\
\hline PSC & 2 & $66.7 \%$ & 1 & $33.3 \%$ & 0 & $0 \%$ & 3 & $100 \%$ & 3 & $100 \%$ & 3 & $8.6 \%$ \\
\hline PMM & 1 & $25 \%$ & 2 & $50 \%$ & 0 & $0 \%$ & 0 & $0 \%$ & 0 & $0 \%$ & 4 & $11.4 \%$ \\
\hline Total & \multicolumn{2}{|c|}{$39(55.7 \%)$} & \multicolumn{2}{|c|}{$28(40 \%)$} & \multicolumn{2}{|c|}{$13(18.6 \%)$} & \multicolumn{2}{|c|}{58 (82.9\%) } & \multicolumn{2}{|c|}{54 (77.1\%) } & \multicolumn{2}{|c|}{$70(100 \%)$} \\
\hline \multirow{2}{*}{ Result } & \multicolumn{2}{|c|}{$X^{2}=9.78$} & \multicolumn{2}{|c|}{$x^{2}=46.66$} & \multicolumn{2}{|c|}{$X^{2}=7.65$} & \multicolumn{2}{|c|}{$X^{2}=3.62$} & \multicolumn{2}{|c|}{$X^{2}=1.29$} & & \\
\hline & \multicolumn{2}{|c|}{${ }^{\star} p=0.002$} & \multicolumn{2}{|c|}{${ }^{*} p=0.001$} & \multicolumn{2}{|c|}{${ }^{*} p=0.006$} & \multicolumn{2}{|c|}{$p=0.057$} & $p=$ & & & \\
\hline
\end{tabular}

\footnotetext{
"significant if $p<0.05$; "None of the tumors react with desmin.
}

marker in tumor groups. PAX8, ER and Ber-EP4 were found to be useful in differential diagnosis between serous carcinomas and peritoneal malignant mesothelioma. WT1 was found to be useful in differential diagnosis between serous carcinomas originated from primary and secondary Müllerian system and CD56 was found to be useful in differential diagnosis of ovarian and tubal serous carcinomas from peritoneal malignant mesotheliomas. 


\section{Discussion}

Nowadays, Müllerian and coelomic hypothesis about the origin of ovarian epithelial tumors are still controversial $[6,8]$. Although it is widely accepted that ovarian epithelial tumours arise in the coelomic epithelium that covers the ovarian surface, researches conducted recently regarding the origin of ovarian cancer has begun to alter the classical information and in view of these findings are that the origin of serous carcinomas is tubal fimbrial epithelium in most cases $[7,8,13]$. The idea that different types of epithelial junctions is considered as hot spots in carcinogenesis, TPJ could be a source of serous carcinomas. But this site cannot account for all of these tumours, some of which are most likely derived from components of the SMS $[6-8,10,13-19]$. In the light of these findings it is assumed that serous carcinomas usually have fimbriated origin, endometrioid and clear cell carcinomas have endometrial origin, whereas Brenner and mucinous tumors may arise from fimbrias by transitional metaplasia $[5,11]$.

OSC, TSC, ESC, PSC, and tubulopapillary pattern of PMM are histologically similar tumors to each other. Panels including a large number of $\mathrm{IHC}$ markers such as p53, WT1, calretinin, D2-40, mesothelin, trombomodulin, CA125, HBME-1, LeuM1, CEA, CK20, CK7, Ber-EP4, CK5/6, $E M A, E R$, and $P R$ are used in differential diagnosis.

In this study, exclusively tumors arising from the Müllerian system and peritoneal mesothelium were considered. Possible relationships of the tumors were investigated according to the expressions of the six IHC markers. In the selection of markers we have taken into account for; the high sensitivity and specificity of PAX8 which is recently identified as a Müllerian determinant; WT1 which is included mesothelioma panel but only recently identified as a determinant of Mullerian; CD56 which is a sensitive marker for neuroendocrine tumors but recently stated to be of diagnostic value for ovarian sex cord stromal tumors, and it has not been studied enough yet in gynecological malignancies. Determinants were also taken into account in the selection of Ber-EP4 as an epithelial, and desmin as a mesenchymal marker to assist the origin of these tumors whether they were epithelial or mesenchymal. Ultimately, ER, especially recently, which plays an important role in the differential diagnosis of PMM and OSC were also included in the study.

Statistical analysis of the expression values of PAX8, WT1, CD56, Ber-EP4, and ER in different tumor groups were found to be significant except desmin $(p<0.05)$. In the literature, there are studies that show the ovarian surface epithelium has both mesenchymal and epithelial features that makes it different than both endometrial or fallopian tube epithelium. Accordingly, the surface epithelium stains with vimentin, $\mathrm{N}$-cadherin, calretinin, HBME1, CK7, and Ber-EP4 but it does not usually stain with CK20, and CA125 [1,3,9]. Besides, combinations of calretinin with Ber-EP4 as a marker of epithelial deter- minant which shows high expression in SC, and with ER that has an important role in the development of genital tract have been reported to be quite useful in the differential diagnosis of OSC with PMM [20-23]. In the study of Mozes, et al. it has been reported that ER reactivity was demonstrated in $64.3 \%$ (36/56 cases) of OSC, $10.7 \%$ (4/37 cases) of ESC and $16.7 \%$ ( $1 / 6$ cases) of TSC, whereas all PSC cases (5 cases) were negative [24]. In particular, since Ber-EP4 is expressed in SCs in high levels, it is an important marker used in the differentiation of SC and PMM [20-22,25]. High rate of ER positivity is observed in OSC. Especially recently, it has an important role in the differential diagnosis of PMM and OSC. In addition, it is often used routinely in the differential diagnosis, due to negative expression in the SCs of female genital tract $[11,26]$. In contrast, EECs are usually positive for ER. Calretinin, Ber-EP4, ER combination is very useful in the differentiation of PMM and a primary or metastatic SC [20-22,2426].

Concerning the Ber-EP4 expression in our study groups when analyzed according to the results; OSC, TSC, PSC, ESC and EEC have been found to be reactive with epithelial markers but PMM has not. In addition, in the evaluation of $\mathrm{IHC}$ staining characteristics of the tumor types defined as SC, EC, MM, epithelial marker Ber-EP4 was determined in $85.7 \%$ of SCs and in $90.4 \%$ of ECs, likewise high reactivity of ER was identified in $80 \%$ of SCs and $83.9 \%$ of ECs. In contrast, all MMs were negative with both of markers. These results can be explained by different epithelial features and estrogen sensitivity of the tumors. In fact, when this difference was evaluated statitically the differences of SC vs MM and of EC vs MM were significant $(p<0.05)$ in terms of Ber-EP4 and ER expressions. On the other hand, IHC staining patterns of tumors that were divided into two groups as PMS and SMS, showed no significant difference for Ber-EP4 and ER ( $p>0.05)$. As a result, the data in this study revealed that Ber-EP4 and ER are useful markers to identify the epithelial origin as well as in the differential diagnosis of PMM and SC similar to the literature data. However, unlike the study of Mozes, et al. [24], in this study ER showed expression rate as high as $75 \%$ of ESC patients, $62.5 \%$ of TSC patients and $100 \%$ of PSC patients. The differences between two studies were thought to be due to the different antibody clones and the small number of ESC, TSC, and PSC cases. In addition, while staining ratio of ER in EEC cases (83.9\%) consistent with the literature, higher staining ratio found than expected in ESC cases (75\%) can be due to the small number of cases in the study.

Desmin is often used in the differential diagnosis of MM with mesothelial hyperplasia because it shows positive staining in reactive mesothelial cells and a very low rate of expression in MM [3,9,27-30]. We couldn't find studies regarding desmin expression in relation to the ovarian surface epithelial tumors. In our study, although PMS and SMS tumors were generally positive with both 
Müllerian (PAX8 and WT1) and epithelial (Ber-EP4) markers, none of them react with desmin. This feature may be an indication of a loss of mesenchymal features in tumor cells. In this study, desmin were used just as a mesenchymal marker. Undoubtedly, to determine the mesenchymal properties of epithelial-stromal tumors of the ovary alone with desmin is not satisfactory alone but evaluation of desmin is thought to be guiding. In this study, all cases of PMM negatively stained with desmin was found consistent with the literature.

Recently, PAX8 has been shown to be a sensitive and specific marker for Müllerian system tumors [3135]. Furthermore, tubal ciliated cells are negative and the secretory cells are stained positive with PAX8 and secretory cells has been suggested as the origin of OSC $[31,34,36]$. In addition, studies noted that PAX8 expression is quite specific in differentiating OSC than MM [31,37]. In this study, the highest rate of PAX8 expression was determined in OSC tumor group (90\%). In addition, PAX8 expression was also determined in TSC, EEC, PSC and even in PMM to a lesser extent but no expression was found in ESC. In pairwise comparisons of tumor groups in terms of PAX8 expresion, differences between OSC with ESC, EEC, and PMM were found to be significant $(p<0.005)$.

WT1, helps the distinction of ESC from SC originating outside the uterus because it is usually negative in ESC but positive in TSC, OSC, and PSC. This difference is linked to genetic differences between tumors [38-44]. In our study we noted that although WT1 was generally positive in SC of fallopian tube, ovarian and peritoneal origin, it didn't show positivity in EEC and ESC. This results showed us that WT1 expression may assist to differentiate ESC from SCs which is originating outside the uterus, in other words from PMS and SMS, but not useful in the differential diagnosis of OSC, PSC, TSC and PMM. In this study, WT1 expression was found as high as PAX8 in OSC (90\%), and found to a lesser extent in TSC, PSC and PMM, but it was not determined in ESC and EEC. When the tumors were divided into three groups as SC, EC and MM according to histological types, and staining characteristics of PAX8 and WT1 were examined statistically, PAX8 expressions were significant in terms of the comparison of SC vs EC and SC vs MM, and WT1 expressions were significant in terms of the comparison of SC vs EC and EC vs MM ( $p<0.05)$. In tumors divided into two groups including PMS and SMS, PAX8 expression was observed in $37.1 \%$ of PMSs and in $74.3 \%$ of SMSs, and in case of WT1, no expression was seen in PMS, while $80 \%$ of SMS tumors showed positivity. When PAX8 and WT1 values of the two groups were statistically evaluated in terms of the value of the PAX8 and WT1 difference was significant ( $p<0.005)$.

Studies associated with CD56 and gynecological malignant tumors has not been observed, except a few publications that may be helpful in differentiating ovarian endometriosis from endometrioid tumors and in diagnostic value in endometrial stromal tumors $[25,45$ -
51]. Working with CD56 in our tumor groups positive staining was detected in OSC (40\%), in TSC (37.5\%), and in EEC (6.5\%), but staining was not detected in ESC, PSC and PMM cases. When the values of CD56 expressions belonging to tumor groups were compared in pairs, the differences of OSC vs EEC and TSC vs EEC were significant $(p<0.05)$. However, divided into three groups according to histological type in terms of CD56 expression, differences between SC vs EC and SC vs MM were significant ( $<<0.05)$, while no significant difference was detected between EC and MM ( $p$ > 0.05). In statistical analysis of the tumors divided into two groups including PMS and SMS in respect to CD56 expression, the difference between PMS and SMS were found as significant $(p<0.005)$. With these results, it can be concluded that CD56 may be a useful marker in the differential diagnosis of OSC with TSC and PMM

Undoubtedly, molecular and genetic studies is necessary in determining the origin of the tumor. In this study, although IHC analysis lack sufficient sensitivity and specificity to determine the mutations, we searched the properties of malignant tumors that develop from peritoneal mesothelium and so far accepted to arise from different localization of Müllerian epithelium by using mesothelial, Müllerian, epithelial and mesenchymal markers as well as their relations with PMS and SMS. It seems possible that TPJ can take place around the fimbriated end of the fallopian tube or ovary, at the same time it seems possible that TPJ could be the junction between PMS and SMS. This junctional area, may have prepared the ground for Müllerian metaplasia as a result of injury occuring in each ovulation due to epithelial degeneration and regeneration processes that has developed by the ovarian and the surrounding area which is similar to formation of cervical squamous metaplasia. It seems that the occurrence of different tumors with the introduction of the different oncogenic pathways which is triggered by different genetic mechanisms might be possible. In our study, we found that IHC features in PMM were different than OSC, TSC and IHC features in ESC were different than OSC and TSC and we also found that epithelial markers were found to be dominant in the tumors except PMM. In particular, although PAX8, WT1 and CD56 were not immunoreactive in ESC, high immunoreactivity in OSC and TSC was considered as an indication which have different origins of the tumors as SMS with PMS. In conclusion TPJ may play an important role in the development of tuboovarian tumors as a junctional place of PMS and SMS. As the tumors of PMS and SMS the tumors arising from peritoneal mesothelium and Müllerian epithelium have different expression immunohistochemically but the finding in the study should be supported by molecular and genetic studies.

\section{Acknowledgements}

The present study was supported by Cumhuriyet University Scientific Research Projects (CUBAP) Project no 
T-501. We are grateful to Ersin Tuncer MD, Serkan Celikgün and Ziynet Cınar for providing us technical supports. This study was presented as oral report at the $23^{\text {rd }}$ National Pathology Congress Çeşme/izmir, TURKEY.

\section{Ethics Committee Approval}

Fundamental ethical approval for the study was taken from Cumhuriyet University School of Medicine Scientific Research Evaluation Committee, with decision number of 2011/018 on the $13^{\text {th }}$ of December, 2011.

\section{Conflicts of Interest and Source of Funding}

The present study was supported by Cumhuriyet University Scientific Research Projects (CUBAP) Project no T-501. The authors declare no conflict of interest.

\section{References}

1. Ellenson LH, Pirog EC (2010) The female genital tract. In: Kumar V, Abbas AK, Fausto N, Aster JC, Robbins and Cotran Pathologic Basis of Disease. ( $8^{\text {th }}$ edn), Saunders Elsevier, Philedelphia, USA, 1005-1063.

2. Al-Nafussi A (2004) Ovarian epithelial tumours: common problems in diagnosis. Current Diagnostic Pathology 10 473-499.

3. Robboy SJ, Mutter GL, Prat J, Bentley RC, Russel P, et al. (2009) Robboy's Pathology of the Female Reproductive Tract. ( $2^{\text {nd }}$ edn), Churchill Livingstone Elsevier, China.

4. Lauchlan SC (1994) The secondary Müllerian system revisited. Int J Gynecol Pathol 13: 73-79.

5. Seidman JD, Yemelyanova A, Zaino RJ, Kurman RJ (2011) The fallopian tube-peritoneal junction: a potential site of carcinogenesis. Int J Gynecol Pathol 30: 4-11.

6. Dubeau L (2008) The cell of origin of ovarian epithelial tumours. Lancet Oncol 9: 1191-1197.

7. Kurman R, Shih IE (2010) The origin and pathogenesis of epithelial ovarian cancer: a proposed unifying theory. Am $J$ Surg Pathol 34: 433-443.

8. Dubeau L (1999) The cell of origin of ovarian epithelial tumors and the ovarian surface epithelium dogma: does the emperor have no clothes? Gynecol Oncol 72: 437-442.

9. Okamato S, Okamato A, Nikaido T, Saito M, Takao M, et al (2009) Mesenchymal to epithelial transition in the human ovarian surface epithelium focusing on inclusion cysts. Oncol Rep 21: 1209-1214.

10. Crum CP, Nucci MR, Lee KR (2011) Diagnostic Gynecologic and Obstetric Pathology. (2 ${ }^{\text {nd }}$ edn), Elsevier Saunders, Philadelphia, USA.

11. Kurman RJ, Ellenson LH, Ronnet BM (2011) Blaunstein's Pathology of the Female Genital Tract. ( $6^{\text {th }}$ edn), New York, USA, Springer.

12. Brustmann H (2005) Immunohistochemical detection of human telomerase reverse transcriptase (hTERT) and c-kit in serous ovarian carcinoma: a clinicopathologic study. Gynecologic Oncology 98: 396-402.

13. Kurman RJ, Shih IM (2011) Molecular pathogenesis and extraovarian origin of epithelial ovarian cancer-Shifting the paradigm. Hum Pathol 42: 918-931.

14. Scully RE (1995) Pathology of ovarian cancer precursors. J Cell Biochem Suppl 23: 208-218.
15. Przbycin CG, Kurman RJ, Ronnett BM, Shih leM, Vang $R$ (2010) Are all pelvic (nonuterine) serous carcinomas of tubal origin? Am J Surg Pathol 34: 1407-1416.

16. Karst AM, Drapkin R (2011) The new face of ovarian cancer modeling: better prospects for detection and treatment. F1000 Med Rep 3: 22.

17. Tong GX, Chiriboga L, Hamele-Bena D, Borczuk AC (2007) Expression of PAX2 in papillary serous carcinoma of the ovary: immunohistochemical evidence of fallopian tube or secondary Müllerian system origin? Mod Pathol 20: 856863.

18. Alison M Karst, Keren Levanon, Ronny Drapkin (2011) Modeling high-grade serous carcinogenesis from the fallopian tube. Proc Natl Acad Sci U S A 108: 7547-7552.

19. Vang R, Shih IM, Kurman R (2009) Ovarian lowgrade and high grade serous carcinoma: pathogenesis clinicopathologic and molecular biologic features and diagnostic problems. Adv Anat Pathol 16: 267-282.

20. Barnetson RJ, Burnett RA, Downie I, Harper CM, Roberts F (2006) Immunohistochemical analysis of peritoneal mesothelioma and primary and secondary serous carcinoma of the peritoneum: antibodies to estrogen and progesterone receptors are useful. Am J Clin Pathol 125: 67-76.

21. Ordóñez NG (2006) Value of immunohistochemistry in distinguishing peritoneal mesothelioma from serous carsinoma of the ovary and peritoneum. a review and update. Adv Anat Pathol 13: 16-25.

22. Comin CE, Saieva C, Messerini L (2007) h-caldesmon, calretinin, estrogen receptor, and Ber-EP4: a useful combination of immunohistochemical markers for differentiating epithelioid peritoneal mesothelioma from serous papillary carcinoma of the ovary. Am J Surg Pathol 31: 1139-1148.

23. Ordóñez NG (2006) The diagnostic utility of immunohistochemistry and electron microscopy in distinguishing between peritoneal mesotheliomas and serous carcinomas: a comparative study. Mod Pathol 19: 34-48.

24. Mozes SN, Khalifa MA, Ismiil N, Saad RS, Hanna WM, et al. (2008) Immunophenotyping of serous carcinoma of the female genital tract. Mod Pathol 21: 1147-1155.

25. Rosai J (2011) Special techniques in surgical pathology. In: Rosai J, Rosai and Ackerman Surgical Pathology. (10 edn), Mosby Elsevier, UK, Edinburgh, 37-94.

26. Soslow RA, Longacre TA (2012) Uterine pathology. (1 ${ }^{\text {st }}$ edn), Cambridge Press New York, USA.

27. Terada T (2011) Immunohistochemical profile of normal mesothelium and histiocytic/ mesothelial hyperplasia: a case report. Int J Clin Exp Pathol 4: 631-636.

28. Husain AN, Colby TV, Ordóñez NG, Krausz T, Borczuk A, et al. (2009) Guidelines for pathologic diagnosis of malignant mesothelioma: a consensus statement from the International Mesothelioma Interest Group. Arch Pathol Lab Med 133: 1317-1331.

29. Attanoos RL, Griffin A, Gibbs AR (2003) The use of immunohistocemistry in distunguishing reactive from neoplastic mesothelium. A novel use for desmin and comparative evaluation with epithelial mebrane antigen, p53, platelet derived growth factor-receptor, P-glycoprotein and $\mathrm{Bcl}-2$. Histopathology 43: 231-238.

30. Auersperg N, Wong AS, Choi KC, Kang SK, Leung PC (2001) Ovarian surface epithelium: biology, endocrinology, and pathology. Endocr Rev 22: 255-288. 
31. Laury AR, Hornick JL, Perets R, Krane JF, Corson J, et al. (2010) PAX8 reliably distinguishes ovarian serous tumors from malignant mesothelioma. Am J Surg Pathol 34: 627635.

32. Lı Xıang, Beıhua Kong (2013) PAX8 is a novel marker for differantiating between various types of tumor, particularly ovarian epithelial carcinomas. Oncol Lett 5: 735-738.

33. Zhai QJ, Ozcan A, Hamilton C, Shen SS, Coffey D, et al (2010) PAX2 expression in non-neoplastic, primary neoplastic, and metastatic neoplastic tissue: A comprehensive immunohistochemical study. Appl Immunohistochem Mol Morphol 18: 323-332.

34. Ozcan A, Shen SS, Hamilton C, Anjana K, Coffey D, et al. (2011) PAX8 expression in non-neoplastic tissues, primary tumors and metastatic tumors: a comprehensive immunohistochemical study. Mod Pathol 24: 751-764.

35. Nonaka D, Chiriboga L, Soslow RA (2008) Expression of PAX8 as a useful marker in distinguishing ovarian carcinomas from mammary carcinomas. Am J Surg Pathol 32: $1566-1571$.

36. Bowen NJ, Logani S, Dickerson EB, Kapa LB, Akhtar M, et al. (2007) Emerging roles for PAX8 in ovarian cancer and endosalpingeal development. Gynecol Oncol 104: 331-337.

37. Mcknight R, Cohen C, Siddiqui MT (2010) Utility of paired box gene 8 (PAX8) expression in fluid and fine-needle aspiration cytology: an immunohistochemical study of metastatic ovarian serous carcinoma. Cancer Cytopathol 118: 298-302.

38. Chivukula M, Dabbs DJ, O'Connor S, Bhargava R (2009) PAX2: a novel Müllerian marker for serous papillary carcinomas to differantiate from micropapillary breast carcinoma. Int J Gynecol Pathol 28: 570-578.

39. Nakatsuka S, Oji Y, Horiuchi T, Kanda T, Kitagawa M, et al. (2006) Immunohistochemical detection of WT1 protein in a variety of cancer cells. Mod Pathol 19: 804-814.

40. Al-Hussaini M, Stockman A, Foster H, McCluggage WG (2004) WT-1 assists in distinguishing ovarian from uterine serous carcinoma and in distinguishing between serous and endometrioid ovarian carcinoma. Histopatholy 44: 109-115.

41. Hashi A, Yuminamochi T, Murata S, Iwamoto $H$, Honda $\mathrm{T}$, et al. (2003) Wilms tumor gene immunoreactivity in primary serous carcinomas of the fallopian tube, ovary, endometrium, and peritoneum. Int J Gynecol Pathol 22: 374-377.
42. Goldstein NS, Uzieblo A (2002) WT1 immunoreactivity in uterine papillary serous carcinomas is different from ovarian serous carcinomas. Am J Clin Pathol 117: 541-545.

43. Egan JA, Ionescu MC, Eapen E, Jones JG, Marshall DS (2004) Diferential expression of WT1 and p53 in serous and endometrioid carcinomas of the endometrium. Int $\mathrm{J}$ Gynecol Pathol 23: 119-122.

44. Cathro HP, Stoler MH (2005) The utility of calretinin, inhibin, and WT1 immunohistochemical staining in the differantial diagnosis of ovarian tumors. Hum Pathol 36: 195-201.

45. He H, Luthringer DJ, Hui P, Lau SK, Weiss LM, et al. (2008) Expression of CD56 and WT1 in ovarian stroma and ovarian stromal tumors. Am J Surg Pathol 32: 884-890.

46. Mccluggage WG, Mckenna M, Mcbride HA (2007) CD56 is a sensitive and diagnosticallay useful immunohistochemical marker of ovarian sex cord-stromal tumors. Int J Gynecol Pathol 26: 322-327.

47. Hans-Ullrich Völker, Sabine Engert, Andreas Cramer, Melanie Schmidt, Ulrike Kämmerer, et al. (2008) Expression of CD56 isoforms in primary and relapsed adult granulosa cell tumors of the ovary. Diagn Pathol 3: 29.

48. Clifford K, Flanagan AM, Regan L (1999) Endometrial CD56+ natural killer cells in women with recurrent miscarriage: a histomorpheometric study. Hum Reprod 14: $2727-2730$

49. Hatice Özer, Handan Aker, Ahmet Mutlu, Ali Yanık, Tülay Koç, et al. (2013) CD56 might be a useful diagnostic marker of müllerian-derived tumors: report of an uterine tumor resembling ovarian sex cord tumor (UTROSCT) suggesting polyphenotypic origin and review of the literature. Basic and Clinical Sciences 2: 132-141.

50. Eun Yoon Cho, YoonLa Choi, Seoung Wan Chae, Jin Hee Sohn, Geung Hwan Ahn (2006) Immunohistochemical study of the expression of adhesion molecules in ovarian serous neoplasms. Pathology International 56: 62-70.

51. Ohishi Y, Kaku T, Oya M, Kobayashi H, Wake N, et al. (2007) CD56 expression in ovarian granulosa cell tumors and its diagnostic utulity and pitfalls. Gynecol Oncol 107: 30-38 